\title{
Changes in $M Y C N$ expression in human neuroblastoma cell lines following cisplatin treatment may not be related to $M Y C N$ copy numbers
}

\author{
PAVEL PROCHAZKA ${ }^{1,2}$, JAN HRABETA $^{1}$, ALES VICHA $^{1}$, SIMON CIPRO $^{1}$, \\ EVA STEJSKALOVA ${ }^{1}$, ZDENEK MUSIL $^{3}$, PAVEL VODICKA ${ }^{2,3}$ and TOMAS ECKSCHLAGER ${ }^{1}$ \\ ${ }^{1}$ Department of Paediatric Hematology and Oncology, Second Medical School, Charles University in Prague \\ and University Hospital Motol, 15006 Prague; ${ }^{2}$ Institute of Experimental Medicine, Academy of Sciences \\ of the Czech Republic, 14200 Prague 4; ${ }^{3}$ Institute of Biology and Medical Genetics, Charles University in \\ Prague, First Faculty of Medicine and General Teaching Hospital, 12108, Prague 2, Czech Republic
}

Received January 17, 2013; Accepted March 13, 2013

DOI: $10.3892 /$ or.2013.2383

\begin{abstract}
Neuroblastoma is a tumor accounting for approximately $10 \%$ of all childhood malignancies and $50 \%$ of all childhood cancer-related deaths. MYCN gene copy number variation represents the most important prognostic factor in neuroblastoma. Prognostic significance of $M Y C N$ gene expression is more complicated and may depend on other factors such as $M Y C N$ gene copy number status. In the present study, we assessed $M Y C N$ gene expression using real-time RT-PCR following cisplatin treatment in three human neuroblastoma cell lines (UKF-NB-3, UKF-NB-4 and SK-N-AS) and their cisplatin-resistant counterparts. We also examined MYCN gene status and copy number (gain and amplification) variations using interphase and metaphase fluorescent in situ hybridization (FISH) and multiplex ligation-dependent probe amplification (MLPA). Only cisplatin-sensitive UKF-NB-4 cells exhibited decreased $M Y C N$ expression following treatment with cisplatin. Other sensitive neuroblastoma cells did not exhibit a change in $M Y C N$ expression. In contrast, cisplatin-resistant UKF-NB-4 and SK-N-AS cells exhibited increased $M Y C N$ expression irrespective of the number of $M Y C N$ copies or concentration of cisplatin in the medium. In $M Y C N$-amplified neuroblastoma cells we did not observe any significant change in the number of $M Y C N$ copies after cisplatin treatment, whereas $M Y C N$-non-amplified SK-N-AS cells revealed during cisplatin treatment an increased number of $M Y C N$ gene copies caused by $2 \mathrm{p}$ gain in the majority of cells by FISH. We postulated that cisplatin treatment does not
\end{abstract}

Correspondence to: Dr Pavel Prochazka, Institute of Experimental Medicine, Academy of Sciences of the Czech Republic, Videnska 1083, 14200 Prague 4, Czech Republic

E-mail:proch.pavel@gmail.com

Key words: high-risk neuroblastoma cell line, multiplex ligationdependent probe amplification, fluorescent in situ hybridization, cisplatin resistance, 2p gain, $M Y C N$ amplification, $M Y C N$ expression result directly in altered transcription of $M Y C N$. A functional change in $M Y C N$ mRNA levels and increased $M Y C N$ expression in cisplatin-resistant neuroblastoma cells do not have a clear relationship to $M Y C N$ copy numbers. These findings may further contribute to the understanding of cisplatin chemotherapy in connection with $M Y C N$ expression, and the possible copy number variations in $M Y C N$ neuroblastoma cells may be of importance since targeting of $M Y C N$ is being tested as neuroblastoma therapy.

\section{Introduction}

Neuroblastoma (NBL), a tumor of the sympathetic nervous system, is the most common solid extracranial childhood tumor, and is responsible for $50 \%$ of all childhood cancer-related deaths. V-myc myelocytomatosis viral-related oncogene, neuroblastoma-derived $(M Y C N)$ was the first amplified oncogene that was found to be of high clinical significance due to its association with the most aggressive form of $\operatorname{NBL}(1,2)$. $M Y C N$ belongs to the family of $M Y C$ genes ( $M Y C, M Y C N$ and $M Y C L$ ) which encode transcription regulators that participate in the control of both cell growth and apoptosis depending on the cellular context (3). In healthy humans, $M Y C N$ is expressed only in certain embryonal tissues, and in adult cells its expression is very low or missing (4). The human $M Y C N$ gene is normally located on the short arm of chromosome 2 (2p24). The term 2p24 gain is suggested as a descriptive generic term for $M Y C N$ signal numbers exceeding up to 4-fold the number of reference signals on the chromosomal arm $2 \mathrm{q}$. This pattern could reflect either a gain of short arms of chromosome $2(2 \mathrm{p}$ gain) or a gain of the $M Y C N$ gene (MYCN gain) which is not an independent prognostic factor in NBL (5) and could indicate an 'incipient' $M Y C N$ amplification (6). $M Y C N$ amplification is defined as a $>4$-fold increase in the $M Y C N$ signal number compared to the reference probe, located on chromosome 2q (6). Amplified $M Y C N$ can take the form of either extrachromosomal double minutes or as homogeneously staining chromosomal regions (HSRs), which are usually located on chromosomes other than 2p (normal MYCN location) (7). 
Three morphologically distinct cell types termed $\mathrm{N}$ (neuroblastic), S (substrate adherent) and I (intermediate) occur in NBL. The biological differences between $\mathrm{S}$ and $\mathrm{N}$ subtypes may be of clinical relevance (8); S-type cells are often prone to develop drug resistance (9). An effective therapy for children with high-risk neuroblastoma (HR NBL) remains one of the greatest challenges for pediatric oncologists. Over the past two decades, numerous attempts have been made to improve the outcomes of these patients by delivering intensive platinum cytostatics based on induction therapy (10). Unfortunately, intensive chemotherapy, which is usually used for treatment of HR NBL, can cause genetic and expression changes in NBL cells. These cellular changes can cause development of drug resistance to the drugs used, thus, ultimately rendering the chemotherapy ineffective. Drug resistance has been described as common in children suffering from NBL, particularly in those whose tumors display amplification and high-level expression of the MYCN oncogene (11). High-level $M Y C N$ expression is associated with unfavorable outcomes and a poor prognosis in tumors with amplified $M Y C N$ (12). However, high-level mRNA $M Y C N$ expression in NBL lacking $M Y C N$ amplification appears to be associated with favorable outcomes (13). High levels of $M Y C N$ mRNA in NBL with $M Y C N$ amplification may be explained by the fact that copies of the amplified gene are actively transcribed (14). Overexpression of the $M Y C$ gene family generally induces unrestricted cell proliferation, inhibition of differentiation, cell growth, angiogenesis, reduced cell adhesion, metastasis and genomic instability (15). MYCN protein has been observed to induce apoptosis in different cells, including NBL and cells with apoptosis induced by cytostatics. NBL cells with $M Y C N$ amplification (and increased gene expression) can resist treatment only when there is additional dysfunction in the apoptotic pathways, such as caspase deficiency (16). MYCN protein can also contribute to the acquired drug-resistance phenotype of cancer cells by modulating the regulation of a specific set of ATP-binding cassette transporter genes to mediate the efflux of chemotherapeutic agents from cancer cells (17).

The aim of our study was to examine $M Y C N$ gene expression in different NBL cell lines, including cisplatin (CDDP)-sensitive or -resistant $\mathrm{N}$ and $\mathrm{S}$ types, following a wide range (100-3,000 ng/ml) of different CDDP doses. The novel aspect of the study includes a comparison of $M Y C N$ gene expression and copies of MYCN gene in NBL cells, following long-term CDDP treatment. MYCN gene copy numbers and its corresponding expression attracted our particular attention since this gene has at the present become a frequent therapeutic target of NBL (18-20).

\section{Materials and methods}

Characteristics of the investigated cell lines. UKF-NB-3 and UKF-NB-4 cell lines with MYCN amplification were established from bone marrow metastases of two patients with HR NBL. The UKF-NB-4 cell line that was established from recurrent disease already possessed the intrinsic multidrug resistance phenotype including 7q21 gain (21). The cell lines were kindly provided by Professor J. Cinatl Jr (Institute for Medical Virology, Hospital of the Johann Wolfgang Goethe University, Frankfurt am Main, Germany). Cells were grown in Iscove's modified Dulbecco's medium (IMDM) with $10 \%$ fetal calf serum (PAA Laboratories, Pasching, Austria). The SK-N-AS cell line was derived from bone marrow metastasis of a female patient with HR NBL. This cell line, which has normal MYCN status, was purchased from the European Collection of Cell Cultures (ECACC, Salisbury, UK) and was cultivated according to the manufacturer's instructions.

The CDDP-resistant counterparts, designated as UKF-NB-3 ${ }^{\text {CDDP }}$ and UKF-NB-4 ${ }^{\text {CDDP, }}$, were also kindly provided by Professor J. Cinatl Jr. SK-N-AS ${ }^{C D D P}$ was prepared in our laboratory by incubation of parental SK-N-AS with increasing concentrations of CDDP either for 14 or 24 months. Solutions of CDDP (Ebewe Pharma Ges.m.b.H. Nfg. KG, Unterach, Austria) were prepared according to the manufacturer's instructions. CDDP-resistant cell lines were cultivated in a medium containing $1 \mu \mathrm{g} / \mathrm{ml}$ of CDDP.

Subsequent CDDP treatment. The above characterized cells were exposed to $100,1,000,2,000$ and 3,000 $\mathrm{ng} / \mathrm{ml}$ CDDP $24 \mathrm{~h}$ after seeding. After $24 \mathrm{~h}$, cells were harvested by trypsinization, washed in sterile phosphate-buffered saline and frozen with TRIzol reagent (Invitrogen, Carlsbad, CA, USA) at $-80^{\circ} \mathrm{C}$ until RNA isolation.

MTT test. The sensitivity of cell lines to CDDP was determined by using the MTT (3-(4,5-dimethylthiazol-2-yl)2,5-diphenyltetrazolium bromide) test (22). The $\mathrm{IC}_{50}$ was assessed using an MTT dye reduction assay as described in a previous report (23). The $\mathrm{IC}_{50}$ values were calculated from at least 3 independent experiments using linear regression of the dose-log response curves using SoftMax Pro microplate data software (Molecular Devices, Sunnyvale, CA, USA).

RNA isolation and real-time reverse transriptase PCR. Total RNA was extracted from all cell lines using TRIzol reagent (Invitrogen). The quality of isolated RNA was verified by horizontal agarose gel electrophoresis, and RNA quantity was measured using a Biomate $3 \mathrm{UV}-\mathrm{V}$ is Spectrophotometer (Thermo Scientific, Waltham, MA, USA). Isolated RNA $(1 \mu \mathrm{g})$ was reverse transcribed to cDNA using random hexamers and a reverse transcription kit (Applied Biosystems, Foster City, CA, USA) according to the manufacturer's instructions. Real-time RT-PCR was assessed using the 7500 Real-Time PCR System (Applied Biosystems) using MYCN and $\beta$-2-microglobulin $(B 2 M)$ primers and probe kits from Generi Biotech (Hradec Kralove, Czech Republic) and TaqMan ${ }^{\circledR}$ Gene Expression Master Mix (Applied Biosystems). Results were expressed using the relative expression software tool (REST) (24) employing $B 2 M$ as the reference gene. $B 2 M$ was chosen for its stable expression at consistent levels in NBL, regardless of clinical stage, and have been used as reference genes in a number of studies of gene expression in NBLs or NBL cell lines (25-27).

Fluorescent in situ hybridization and karyotype. Interphase fluorescent in situ hybridization (FISH) analyses were performed using an ON MYCN (2p24)/LAF (Kreatech Biotechnology B.V., Amsterdam, The Netherlands) and by using Vysis LSI N-MYC (2p24) SpectrumOrange Probe (Vysis, Downers Grove, IL, USA) simultaneously with Alpha 
Satellite 2 Green Probe (Cytocell Technologies, Cambridge, $\mathrm{UK})$, in accordance with the manufacturer's instructions. The use of a 2 p-specific probe simultaneously with $2 \mathrm{q}$ signals is recommended by the International Neuroblastoma Risk Group Biology Committee (6). Slides were scored with an Olympus AX70 (Olympus Optical Co., Ltd., Japan) fluorescence microscope with a CCD camera (Jenoptik, Jena, Germany). Images of the interphase nuclei were digitally captured using an appropriate software program (MetaSystems, Altlussheim, Germany). Two hundred nuclei were investigated from each cell line as recommended for evaluation by FISH analyses (28).

For karyotype analysis, exponentially growing cells cultivated in medium without CDDP were harvested with trypsin, treated with hypotonic solution, and fixed with 3:1 methanol/acetic acid. Slides were banded using standard trypsin-Giemsa banding (GTG-banding) or were used for metaphase FISH analysis performed by Vysis LSI N-MYC (2p24) SpectrumOrange Probe simultaneously with Alpha Satellite 2 Green Probe in accordance with the manufacturer's instructions.

Multiplex ligation-dependent probe amplification. Multiplex ligation-dependent probe amplification (MLPA) analysis was performed to identify whether the region gained or amplified included adjacent genes in addition to the MYCN gene. We used the neuroblastoma-specific SALSA MLPA P252B Probe Kit (MRC-Holland, Amsterdam, The Netherlands) which analyzes chromosomes 2 and 17. Detailed information on probe sequences, gene loci and chromosome locations can be found at www.mlpa.com. Genomic DNA (50-200 ng) was denatured and the probes were allowed to hybridize (16 h at $60^{\circ} \mathrm{C}$ ). PCR was performed on the samples in a volume of $50 \mu \mathrm{l}$ containing $10 \mu \mathrm{l}$ of the ligation reaction mixture and using a thermal cycler Mastercycler ep gradient (Eppendorf, Hamburg, Germany). Aliquots of $1 \mu \mathrm{l}$ of the PCR reaction were combined with $0.5 \mu \mathrm{l}$ ROX-labeled internal size standard (Applied Biosystems) and $12 \mu \mathrm{l}$ deionized formamide. Fragments were separated by electrophoresis on an Applied 3130xl capillary sequencer and quantified using the intermediate version Coffalyser (MRC Holland). For copy-number detection, four normal control DNA samples were included in each set of MLPA experiments. Interpretation was based on the comparison of peak heights between the control DNA and the tumor sample.

Statistical analysis. All numerical data are presented as means \pm standard deviation and analyzed statistically using the Student's t-test; mRNA expression results were analyzed using REST software (24).

\section{Results}

The sensitivity of cell lines to CDDP. The sensitivity of the cell lines to CDDP was verified using the MTT test. The resistant cell lines showed at least a 2 -fold increase in resistance to CDDP measured as $\mathrm{IC}_{50}$. The data presented in Table I indicated the UKF-NB-3 cell line as the most sensitive and SK-N-AS ${ }^{\mathrm{CDDP}}$ and UKF-NB-4 ${ }^{\mathrm{CDDP}}$ as the most resistant towards CDDP.
Table I. Levels of the drug-resistance of the tested NBL cell lines expressed as $\mathrm{IC}_{50}$.

\begin{tabular}{lcc}
\hline Cell line & NBL type & $\mathrm{IC}_{50}(\mu \mathrm{M})$ CDDP \\
\hline SK-N-AS & $\mathrm{S}$ & $3.71 \pm 0.67$ \\
SK-N-ASC ${ }^{\text {CDDP }}$ & $\mathrm{S}$ & $11.68 \pm 2.84$ \\
UKF-NB-3 $^{\text {CKF-NB-3 }}$ CDDP & $\mathrm{N}$ & $0.99 \pm 0.01$ \\
UKF-NB-4 $_{\text {UKF-NB-4 }}^{\text {CDDP }}$ & $\mathrm{N}$ & $7.68 \pm 1.37$ \\
UKF $^{-}$ & $\mathrm{S}$ & $4.60 \pm 0.2$ \\
\hline
\end{tabular}

$\mathrm{IC}_{50}$ values were calculated from the linear regression of the dose-log response curves after a 96-h exposure to CDDP, as determined by the MTT assay. Values are the means \pm SD of at least 3 independent experiments. S, S-type of NBL; N, N-type of NBL.

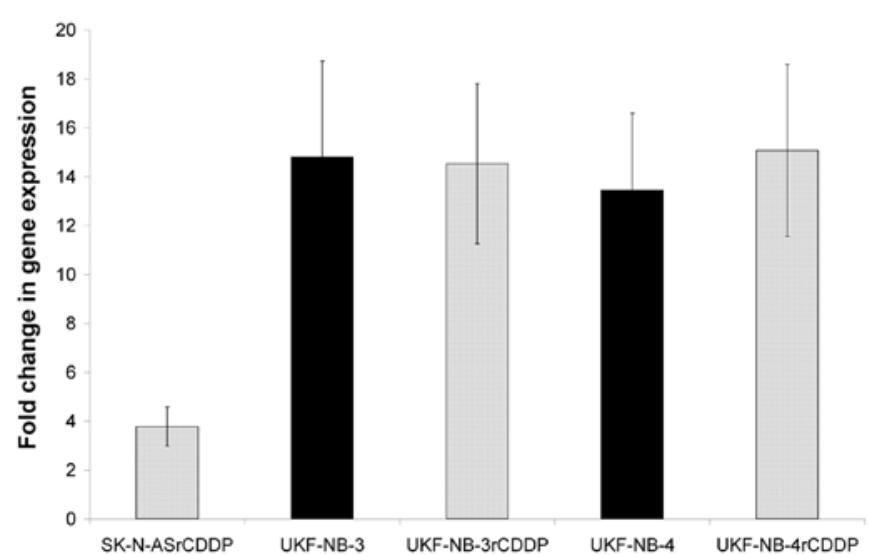

Figure 1. MYCN expression in NBL cell lines and their CDDP-resistant (rCDDP) counterparts. The data are expressed as relative expression levels in comparison with the reference NBL cell line SK-N-AS. Expression analysis of reference gene $\beta$-2-microglobulin was carried out parallel to all experiments. mRNA levels were not different in any expression analysis for the particular cell line.

MYCN expression levels in CDDP-sensitive and -resistant $N B L$ cell lines. Both $M Y C N$-amplified UKF-NB-3 and UKF-NB-4 cell lines had significantly higher $M Y C N$ mRNA expression as compared to the MYCN non-amplified line SK-N-AS (14.8, respectively 13.4-fold higher in 2-log scale, $\mathrm{P}<0.001$ ) (Fig. 1). SK-N-AS ${ }^{\mathrm{CDDP}}$ had 3.8-fold higher expression of MYCN $(\mathrm{P}<0.01)$ than SK-N-AS. We did not find any distinguishable change in $M Y C N$ expression between CDDPsensitive UKF-NB-3 and its CDDP-resistant counterpart (Fig. 1), while UKF-NB-4 ${ }^{\mathrm{CDDP}}$ had a 1.6 -fold higher expression of MYCN $(\mathrm{P}<0.05)$ in comparison to that in the sensitive UKF-NB-4 cell line. There were no significant differences in MYCN expression between UKF-NB-3 and UKF-NB-4 cells nor between UKF-NB-3 ${ }^{\mathrm{CDDP}}$ and UKF-NB-4 ${ }^{\mathrm{CDDP}}$ cell lines. Regarding the CDDP-resistant cell lines, the highest $M Y C N$ expression in comparison with SK-N-AS ${ }^{\mathrm{CDDP}}$ was found in the UKF-NB-4 ${ }^{\mathrm{CDDP}}$ cell line (11.3-fold higher; $\mathrm{P}<0.01$ ), followed by UKF-NB-3 ${ }^{\mathrm{CDDP}}$ (10.8-fold higher; $\left.\mathrm{P}<0.01\right)$.

MYCN expression levels in CDDP-sensitive and -resistant NBL cells following short-term CDDP treatment. CDDP- 

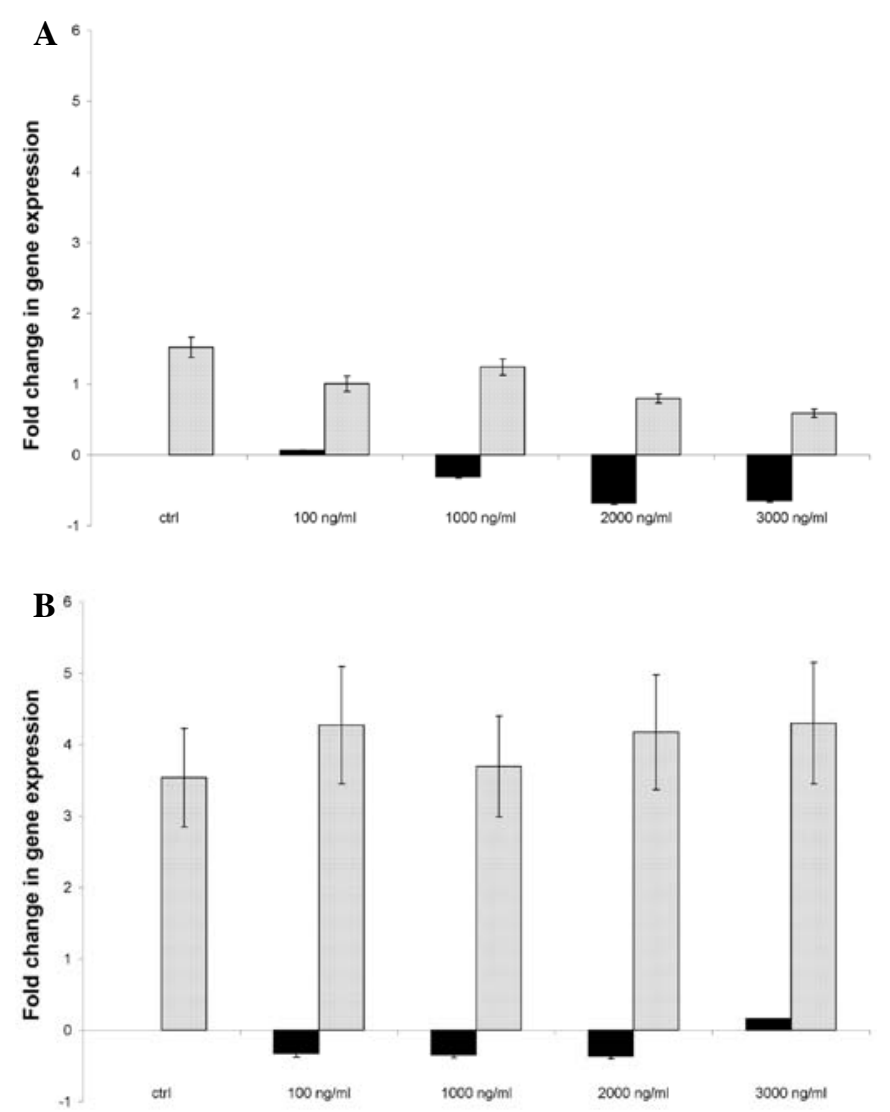

Figure 2. (A) $M Y C N$ expression in UKF-NB-4 and UKF-NB-4 ${ }^{\text {CDDP }}$ cells after treatment with CDDP. CDDP-sensitive-maternal UKF-NB-4 cells are represented by black bars, CDDP-resistant UKF-NB-4 cells are represented by gray bars. The dose of CDDP is depicted on the x-axis. Untreated CDDPsensitive UKF-NB-4 cells were used as a reference value. Each experiment was repeated three times with concordant outcomes. (B) $M Y C N$ expression in SK-N-AS and SK-N-AS ${ }^{\text {CDDP }}$ cells after treatment with CDDP. CDDPsensitive-maternal MYCN non-amplified SK-N-AS cells are represented by black bars, CDDP-resistant SK-N-AS cells are represented by gray bars. The dose of CDDP is depicted on the x-axis. Untreated CDDP-sensitive SK-N-AS cells were used as a reference value. The experiment was repeated twice.

sensitive UKF-NB-4 cells showed a significant decrease in $M Y C N$ expression after cultivation with CDDP concentrations of $1000 \mathrm{ng} / \mathrm{ml}$ and higher ( $\mathrm{P}=0.001)$ (Fig. 2A). CDDP-resistant UKF-NB-4 and SK-N-AS cells showed significant upregulation of $M Y C N$ expression $(\mathrm{P}=0.001)$ in comparison with the sensitive cells, however, a single CDDP dose did not significantly modulate $M Y C N$ expression levels in the resistant cell lines (Fig. 2). These expression changes were not associated with senescence, as determined by staining of investigated cell cultures for the senescence-associated $\beta$-galactosidase marker (data not shown).

In the sensitive SK-N-AS cells, we did not observe any significant changes in MYCN expression after cultivation with any CDDP dose $(\mathrm{P}>0.3)$. The SK-N-AS ${ }^{\mathrm{CDDP}}$ cell line showed a significant increase in $M Y C N$ expression relative to the control maternal line independent of the presence or absence of CDDP in the medium $(\mathrm{P}<0.05)$ (Fig. 2B).

MYCN status in CDDP-treated NBL cells. Using FISH, we observed $M Y C N$ amplification in all 200 investigated nuclei of UKF-NB-3 and UKF-NB-4 cells and their CDDP-resistant counterparts (Fig. 3A and B). UKF-NB-3 and UKF-NB-4
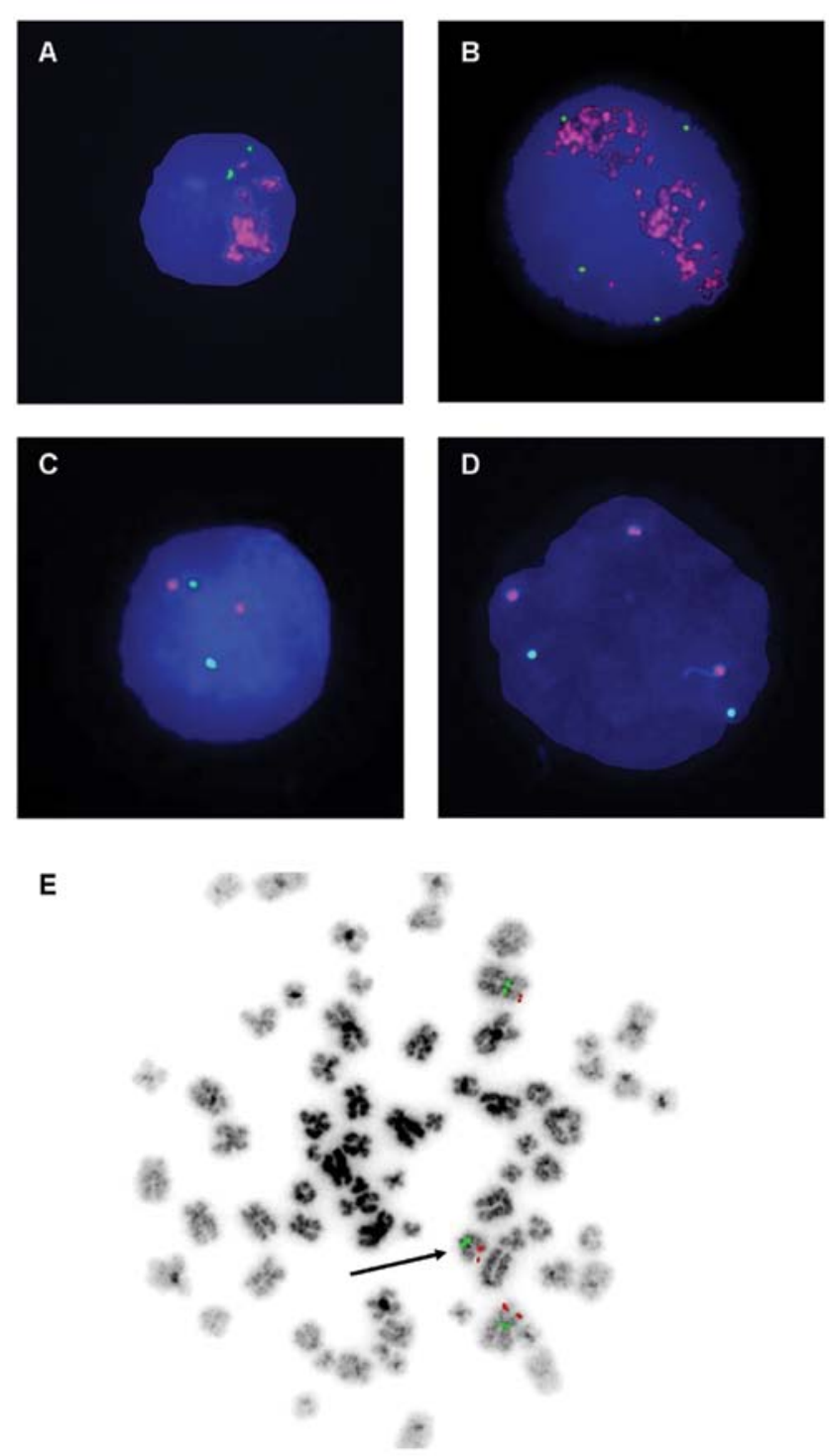

Figure 3. MYCN gene in tested NBL cell lines. FISH displays $M Y C N$ amplification (red signals) in nuclei of (A) UKF-NB-3 and (B) UKF-NB-4 cells (C) The SK-N-AS cell line has two copies of the MYCN gene and two chromosomes 2 (green signals). (D) In the CDDP-resistant SK-N-AS cells, the nuclei exhibited an extra copy of the $M Y C N$ gene (red signals) representing either $2 \mathrm{p}$ gain or $M Y C N$ gain or both. FISH analyses were performed using ON MYCN (2p24)/LAF probes (original magnification, x100). (E) Mitotic chromosomes of SK-N-AS ${ }^{\mathrm{CDDP}}$ cells showed an extra copy of the short arm of chromosome 2 (highlighted by the black arrow) containing the MYCN gene (red dots) and an extra copy of centromere (green dots) representing 2p gain. FISH was performed using LSI N-MYC (2p24) and chromosome 2 Alpha satellite probes (original magnification, $\mathrm{x} 100$ ).

amplified $M Y C N$ in the form of HSRs. Regarding the UKF-NB-3 cells, multiplex ligation-dependent probe amplification (MLPA) revealed disomy of chromosome 2 without any other genetic aberration in 2p except for $M Y C N$ amplification. In UKF-NB-3 ${ }^{\text {CDDP }}$ cells we found a loss of the $2 \mathrm{p} 11.2-2 \mathrm{p} 25.3$ region except for 2p23.2 (ALK) and $M Y C N$-amplified copies. Both CDDP-sensitive and -resistant UKF-NB-4 cells exhibited an amplified 2p24.3 region (NAG, DDX1 and $M Y C N$ genes), but without any other significant change in $2 \mathrm{p}$.

Using interphase FISH analysis with two different sets of probes, the MYCN-non-amplified SK-N-AS cell line showed 
A

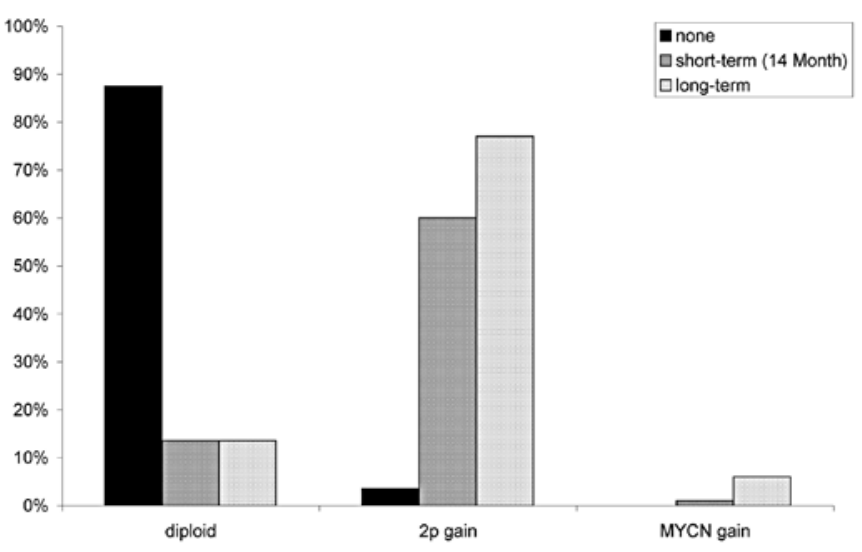

B

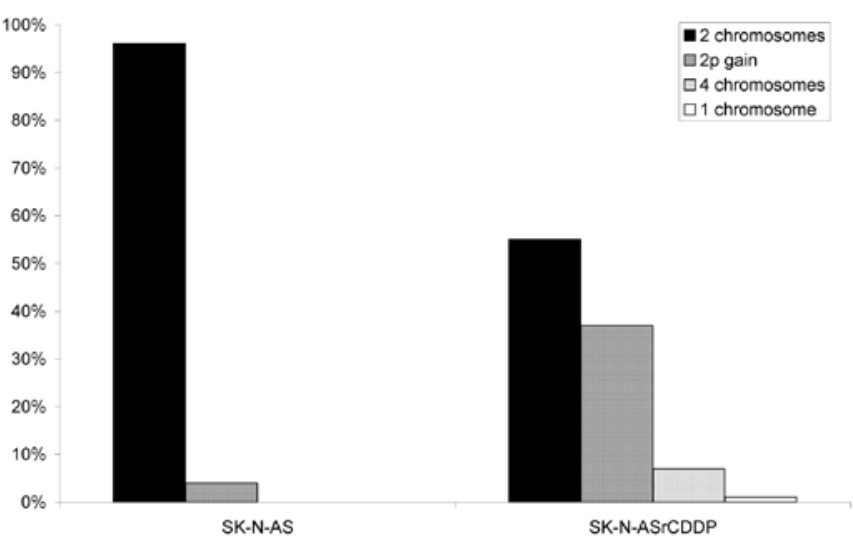

Figure 4. (A) The number of chromosome 2 and percentage of cells with $M Y C N$ gain in the SK-N-AS cell lines follows cultivation with CDDP. For the assessment, 200 interphase nuclei of the tested cell line were scored. Three signals of the LSI N-MYC (2p24) in relation to 3 signals of the chromosome 2 Alpha satelite probe were evaluated as $2 p$ gain. More than 3 signals of $2 p 24$ were evaluated as $M Y C N$ gain. (B) The number of chromosome 2 in mitotic SK-N-AS cells. In each mitosis the number of chromosome 2 was scored and expressed as a percentage of cells with normal and abnormal number of chromosome 2. The type of chromosome 2 gain/loss is also indicated. Investigated cells were prepared by typical cytogenetic preparation using colcemid for metaphase observation. Analyses were performed using LSI N-MYC (2p24) and chromosome 2 Alpha satellite probes.

normal MYCN status in the majority of cells (Fig. 3C) with a sporadically $(3.5 \%)$ occurring clone having an extra $2 p$ (Fig. 4A). After short-term (14 months) and long-term (24 months) cultivation with CDDP, SK-N-AS ${ }^{\text {CDDP }}$ cells were examined again for the $M Y C N$ gene status and for the number of chromosome 2 . The CDDP cultivation resulted in a substantial clonal expansion of cells with $2 \mathrm{p}$ gain, since the SK-N-AS ${ }^{\text {CDDP }}$ cells had 60 and $77 \%$ of cells with three $2 p$ (Fig. 3D) after short-term and long-term cultivation, respectively (Fig. 4A). In contrast, using MLPA we did not find any significant $2 \mathrm{p}$ change in SK-N-AS ${ }^{\mathrm{CDDP}}$ cells, but MLPA analyses whole genomic material regardless of heterogeneity in the cell population as in FISH.

Metaphase FISH analyses of SK-N-AS cells also revealed a predominant occurrence of cells with two chromosomes 2 in mitotic cell and a subsequent increase in cells with $2 p$ gain after CDDP treatment was noted (Fig. 3E). We also observed cells with tetrasomy or monosomy, but their proportion was rather minor (Fig. 4B).

\section{Discussion}

In the present study, 6 NBL cell lines (4 with $M Y C N$ amplification and 2 cell lines without $M Y C N$ amplification) were analyzed. The cell line SK-N-AS ${ }^{\mathrm{CDDP}}$ (without $M Y C N$ amplification), which may serve as a model for the development of drug-resistant NBL, was established and characterized in our laboratory. In the context of CDDP-based NBL therapy, we investigated mRNA $M Y C N$ expression and $M Y C N$ gene status. In the SK-N-AS and SK-N-AS ${ }^{\text {CDDP }}$ cells we found a clear link between the number of $M Y C N$ gene copies and their expression. This finding was in agreement with the study of Valent et al (29) who found higher MYCN expression in three of four NBLs with 2p/MYCN gain in comparison with the SK-N-SH cell line that has $1 M Y C N$ copy in the haploid genome. The cells with high-level MYCN amplification (UKFNB-3, UKF-NB-4 and their CDDP-resistant counterparts) exhibit several-fold higher mRNA $M Y C N$ expression than those without $M Y C N$ amplification. In the case of NBL cell lines with normal number of $M Y C N$ copies and in NBL cell lines with one extra $M Y C N$ copy, the difference in $M Y C \mathrm{~N}$ expression was significant. The biological and clinical characteristics of $2 \mathrm{p} / M Y C N$ gain have not been to date as clearly defined as $M Y C N$ amplification (30). Approximately $10 \%$ of diagnosed NBLs could contain an MYCN gain $(30,31)$. Patients with $M Y C N$ gain demonstated a significantly higher age at diagnosis and according to several studies are associated with an advanced stage as compared to $M Y C N$-normal cases $(30,31)$. In contrast to the study of Spitz et al (31) who reported that NBLs with $M Y C N$ gain displayed no increase in $M Y C N$ mRNA expression, and in agreement with Valent et al (29) who found in the majority of NBLs cases a $2 \mathrm{p} / M Y C N$ gain increased expression, we found a tendency of $M Y C N$ gain and higher MYCN expression in the SK-N-AS ${ }^{\text {CDDP }}$ cells.

Numeric changes of $2 p$ in SK-N-AS cells may suggest the selection advantage of cells with $2 \mathrm{p}$ gain in the presence of CDDP. The simultaneous coexistence of both cell types, cells with disomy of chromosome 2 and cells with 2 p gain, is documented in Fig. 4B. The subsequent clonal expansion of cells with $2 \mathrm{p}$ gain in the presence of CDDP may be a realistic interpretation of this phenomenon as described by Cohen et al (32) in the case of the SK-N-SH cell line. Apparently, senescence has no effect on $2 \mathrm{p}$ gain or increased $M Y C N$ expression in SK-N-AS ${ }^{C D D P}$ cells. Notably, observations based on metaphase FISH analyses of SK-N-AS ${ }^{\text {CDDP }}$ cells (revealing cells with 2 p gain, sporadic tetrasomy or monosomy) have not been confirmed by MLPA analysis, which did not confirm any significant $2 p$ change in both SK-N-AS or SK-N-AS ${ }^{\text {CDDP }}$ cell lines. This discrepancy may be due to the nature of the two techniques: FISH may be more subjective due to the visual evaluation, while capable of taking into account the heterogeneity in the cell population, wherease MLPA, an accurate and sensitive technique, analyzes whole genomic material irrespective of the situation in particular cells (33). Our data suggest the need to analyze amplification of the genetic material by two independent methods in order to avoid false interpretations. 
In addition to clinical (clinical stage, age), biochemical (LDH levels) and genetic (MYCN amplification, 1p36 deletion) prognostic parameters, the expression of several genes have also been reported to predict the outcome of NBL. Overexpressed genes encoding tyrosine kinase receptors (TrkA and EPHB6) and cell surface molecules (CD44, EFNB2, and EFNB3) have been described as favorable markers (13), but the significance of $M Y C N$ expression remains controversial since the survival of children suffering from NBL does not correlate with its expression $(13,34,35)$. A high expression of $M Y C N$ in NBL cells may be associated with both a favorable (cells without $M Y C N$ amplification) and adverse prognosis (cells with amplified $M Y C N$ ) (12). Similarly, $M Y C$ overexpression without amplification has also been linked to a more favorable prognosis in patients with breast (36) and colorectal cancers (37). Better outcomes of patients with tumors that overexpress $M Y C$ or $M Y C N$ are due to either increased apoptosis or enhanced drug-sensitivity evoked by higher proliferation (35). Clinical observation has also been confirmed in in vitro experiments. Transfection of cells with a vector containing MYCN significantly decreased the viability of NBL cells without $M Y C N$ amplification. $M Y C N$ protein induced apoptosis and enhanced expression of genes that were prognostically favorable (13). In vitro studies using an NBL cell line with a controlled $M Y C N$ expression system showed that NBL cells with induced $M Y C N$ expression have higher cytostatically induced apoptosis than NBL cells without induction of $M Y C N$ expression. Cytostatic drugs with various mechanisms of action including CDDP have been tested. All tested compounds were more effective in cells overexpressing $M Y C N$, except for betulinic acid, which induces apoptosis by direct effects on mitochondria (38). The above-mentioned results reporting equal or increased $M Y C N$ expression in CDDP-resistant cell seem to differ from our findings. An explanation may be that the induction of multiple changes in the genome of resistant cells indicates that cytostatics induce drug-resistance through multiple mechanisms $(21,39)$. It is possible that NBL cells without amplification of the $M Y C N$ used in the above mentioned study (38) have normally functioning apoptotic pathways, but in relation to drug-resistance, there is a defect in one of these pathways, possibly due to increased $M Y C N$ expression. Van Noesel et al (40) analyzed a panel of NBL cell lines with $M Y C N$ amplification and showed that $M Y C N$ induces both caspase-8- and caspase-9-mediated apoptosis The authors concluded that an epigenetic downregulation of caspase- 8 in NBLs is unlikely to be induced by overexpression of $M Y C N$. Additional defects in apoptosis may be involved in the caspase-9 route to apoptosis. It appears that defects in apoptotic routes in NBL tumors do have a neutralizing effect on MYCN (40).

As an in vitro model of drug-resistance (22), we chose CDDP-resistant sublines prepared by incubation of parental NBL cells with increasing concentrations of CDDP. We found that the NBL cell line, with predominantly normal status of the MYCN gene, had increased numbers of cells with $2 \mathrm{p}$ gain and increased $M Y C N$ expression after CDDP treatment. Wasenius et al (41) described both deletion and gain of $2 \mathrm{p}$ in CDDP-resistant ovarian cancer cell lines but our finding of $2 p$ gain in NBL in connection with drug-resistance was described for the first time here. We also studied MYCN expression in
CDDP-sensitive and -resistant NBL cells after exposure to CDDP as an in vitro model of chemotherapy. MYCN amplified cell lines, cultivated with CDDP, did not change $M Y C N$ expression. Therefore, cisplatin resistance does not result in a functional change to MYCN mRNA levels. Our findings contribute to the understanding of the influence of chemotherapy on $M Y C N$ expression in NBL cell lines. This is of particular importance since targeting the $M Y C N$ gene may be a therapeutic alternative for NBL (42).

\section{Acknowledgements}

We would like to thank Professor J. Cinatl Jr (Institute for Medical Virology, Hospital of the Johann Wolfgang Goethe University, Frankfurt/Main, Germany) for providing the UKF-NB-3 and UKF-NB-4 cell lines and their CDDPresistant sublines. We also thank Alessio Naccarati (Institute of Experimental Medicine, Academy of Sciences of the Czech Republic) for his critical review. This research was supported by the Grant Agency of the Czech Republic (grant P301/10/0356) and by the project for conceptual development of research organization 00064203.

\section{References}

1. Schwab M: $M Y C N$ in neuronal tumours. Cancer Lett 204: 179-187, 2004.

2. Kyo Y, Tanaka T, Hayashi K, et al: Identification of therapysensitive and therapy-resistant neuroblastoma subtypes in stages III, IVs and IV. Cancer Lett 306: 27-33, 2011.

3. Evan GI and Vousden KH: Proliferation, cell cycle and apoptosis in cancer. Nature 411: 342-348, 2001.

4. Westermark UK, Wilhelm M, Frenzel A and Henriksson MA: The $M Y C N$ oncogene and differentiation in neuroblastoma. Semin Cancer Biol 21: 256-266, 2011.

5. Souzaki R, Tajiri T, Teshiba R, et al: The genetic and clinical significance of MYCN gain as detected by FISH in neuroblastoma. Pediatr Surg Int 27: 231-236, 2011.

6. Ambros PF, Ambros IM, Brodeur GM, et al: International consensus for neuroblastoma molecular diagnostics: report from the International Neuroblastoma Risk Group (INRG) Biology Committee. Br J Cancer 100: 1471-1482, 2009.

7. Prochazka P, Hrabeta J, Vicha A and Eckschlager T: Expulsion of amplified MYCN from homogenously staining chromosomal regions in neuroblastoma cell lines after cultivation with cisplatin, doxorubicin, hydroxyurea, and vincristine. Cancer Genet Cytogenet 196: 96-104, 2010.

8. Baumann Kubetzko FB, Di Paolo C, Maag C, et al: The PAX5 oncogene is expressed in N-type neuroblastoma cells and increases tumorigenicity of a S-type cell line. Carcinogenesis 25: 1839-1846, 2004.

9. Bian X, Giordano TD, Lin HJ, Solomon G, Castle VP and Opipari AW Jr: Chemotherapy-induced apoptosis of S-type neuroblastoma cells requires caspase- 9 and is augmented by CD95/Fas stimulation. J Biol Chem 279: 4663-4669, 2004.

10. Maris JM, Hogarty MD, Bagatell R and Cohn SL: Neuroblastoma. Lancet 369: 2106-2120, 2007.

11. de Cremoux P, Jourdan-Da-Silva N, Couturier J, et al: Role of chemotherapy resistance genes in outcome of neuroblastoma. Pediatr Blood Cancer 48: 311-317, 2007.

12. Brodeur GM: Neuroblastoma: biological insights into a clinical enigma. Nat Rev Cancer 3: 203-216, 2003.

13. Tang XX, Zhao H, Kung B, et al: The MYCN enigma: significance of $M Y C N$ expression in neuroblastoma. Cancer Res 66: 2826-2833, 2006.

14. Lutz W and Schwab M: In vivo regulation of single copy and amplified N-myc in human neuroblastoma cells. Oncogene 15: 303-315, 1997.

15. Westermann F, Muth D, Benner A, et al: Distinct transcriptional MYCN/c-MYC activities are associated with spontaneous regression or malignant progression in neuroblastomas. Genome Biol 9: R150, 2008. 
16. Fulda S, Lutz W, Schwab M and Debatin KM: MycN sensitizes neuroblastoma cells for drug-induced apoptosis. Oncogene 18: 1479-1486, 1999.

17. Porro A, Haber M, Diolaiti D, et al: Direct and coordinate regulation of ATP-binding cassette transporter genes by Myc factors generates specific transcription signatures that significantly affect the chemoresistance phenotype of cancer cells. J Biol Chem 285: 19532-19543, 2010.

18. Bell E, Chen L, Liu T, Marshall GM, Lunec J and Tweddle DA MYCN oncoprotein targets and their therapeutic potential. Cancer Lett 293: 144-157, 2010.

19. Albihn A, Johnsen JI and Henriksson MA: MYC in oncogenesis and as a target for cancer therapies. Adv Cancer Res 107: $163-224,2010$

20. Gustafson WC and Weiss WA: Myc proteins as therapeutic targets. Oncogene 29: 1249-1259, 2010.

21. Prochazka P, Libra A, Zemanova Z, et al: Mechanisms of ellipticine-mediated resistance in UKF-NB-4 neuroblastoma cells Cancer Sci 103: 334-341, 2012.

22. Kotchetkov R, Cinatl J, Blaheta R, et al: Development of resistance to vincristine and doxorubicin in neuroblastoma alters malignant properties and induces additional karyotype changes: a preclinical model. Int J Cancer 104: 36-43, 2003.

23. Poljakova J, Eckschlager T, Hrabeta J, et al: The mechanism of cytotoxicity and DNA adduct formation by the anticancer drug ellipticine in human neuroblastoma cells. Biochem Pharmacol 77: 1466-1479, 2009.

24. Pfaffl MW, Horgan GW and Dempfle L: Relative expression software tool (REST) for group-wise comparison and statistical analysis of relative expression results in real-time PCR. Nucleic Acids Res 30: e36, 2002.

25. Bordow SB, Haber M, Madafiglio J, Cheung B, Marshall GM and Norris MD: Expression of the multidrug resistance-associated protein (MRP) gene correlates with amplification and overexpression of the N-myc oncogene in childhood neuroblastoma. Cancer Res 54: 5036-5040, 1994.

26. Beiske K, Ambros PF, Burchill SA, Cheung IY and Swerts K: Detecting minimal residual disease in neuroblastoma patients the present state of the art. Cancer Lett 228: 229-240, 2005

27. Hogarty MD, Norris MD, Davis K, et al: $O D C 1$ is a critical determinant of $M Y C N$ oncogenesis and a therapeutic target in neuroblastoma. Cancer Res 68: 9735-9745, 2008.

28. Ambros PF and Ambros IM; SIOP Europe Neuroblastoma Pathology, Biology and Bone Marrow Group. Pathology and biology guidelines for resectable and unresectable neuroblastic tumors and bone marrow examination guidelines. Med Pediatr Oncol 37: 492-504, 2001.

29. Valent A, Le Roux G, Barrois M, et al: MYCN gene overrepresentation detected in primary neuroblastoma tumour cells without amplification. J Pathol 198: 495-501, 2002.
30. Jeison M, Ash S, Halevy-Berko G, et al: 2p24 Gain region harboring MYCN gene compared with MYCN amplified and nonamplified neuroblastoma: biological and clinical characteristics. Am J Pathol 176: 2616-2625, 2010.

31. Spitz R, Hero B, Skowron M, Ernestus K and Berthold F: $M Y C N-$ status in neuroblastoma: characteristics of tumours showing amplification, gain, and non-amplification. Eur J Cancer 40: 2753-2759, 2004

32. Cohen N, Betts DR, Rechavi G, Amariglio N and Trakhtenbrot L: Clonal expansion and not cell interconversion is the basis for the neuroblast and nonneuronal types of the SK-N-SH neuroblastoma cell line. Cancer Genet Cytogenet 143: 80-84, 2003.

33. Ambros IM, Brunner B, Aigner G, et al: A multilocus technique for risk evaluation of patients with neuroblastoma. Clin Cancer Res 17: 792-804, 2011.

34. Chan HS, Gallie BL, DeBoer G, et al: $M Y C N$ protein expression as a predictor of neuroblastoma prognosis. Clin Cancer Res 3: 1699-1706, 1997.

35. Cohn SL, London WB, Huang D, et al: MYCN expression is not prognostic of adverse outcome in advanced-stage neuroblastoma with nonamplified MYCN. J Clin Oncol 18: 3604-3613, 2000.

36. Bieche I, Laurendeau I, Tozlu S, et al: Quantitation of $M Y C$ gene expression in sporadic breast tumors with a real-time reverse transcription-PCR assay. Cancer Res 59: 2759-2765, 1999.

37. Smith DR and Goh HS: Overexpression of the c-myc protooncogene in colorectal carcinoma is associated with a reduced mortality that is abrogated by point mutation of the p53 tumor suppressor gene. Clin Cancer Res 2: 1049-1053, 1996.

38. Paffhausen T, Schwab M and Westermann F: Targeted MYCN expression affects cytotoxic potential of chemotherapeutic drugs in neuroblastoma cells. Cancer Lett 250: 17-24, 2007.

39. Bedrnicek J, Vicha A, Jarosova M, et al: Characterization of drug-resistant neuroblastoma cell lines by comparative genomic hybridization. Neoplasma 52: 415-419, 2005.

40. van Noesel MM, Pieters R, Voute PA and Versteeg R: The N-myc paradox: N-myc overexpression in neuroblastomas is associated with sensitivity as well as resistance to apoptosis. Cancer Lett 197: 165-172, 2003.

41. Wasenius VM, Jekunen A, Monni O, et al: Comparative genomic hybridization analysis of chromosomal changes occurring during development of acquired resistance to cisplatin in human ovarian carcinoma cells. Genes Chromosomes Cancer 18: 286-291, 1997.

42. Nara K, Kusafuka T, Yoneda A, Oue T, Sangkhathat S and Fukuzawa M: Silencing of $M Y C N$ by RNA interference induces growth inhibition, apoptotic activity and cell differentiation in a neuroblastoma cell line with $M Y C N$ amplification. Int J Oncol 30: 1189-1196, 2007 